\title{
Spectral Density Functionals for Electronic Structure Calculations
}

\author{
S. Y. Savrasov and G. Kotliar \\ Center for Materials Theory and Department of Physics and Astronomy, Rutgers \\ University, Piscataway, NJ 08854-8019
}

(May 2001)

\begin{abstract}
We introduce a functional of the local spectral electron density which can be used to to compute the total energy and the local spectral function of strongly-correlated materials. We illustrate the applicability of the method by using as an example the long-standing problem of the electronic structure of metallic plutonium.
\end{abstract}

Electronic structure approaches based on density functional theory (DFT) in its local density (LDA) and generalized gradient (GGA) approximations [1] have successfully predicted the ground-state properties of weaklycorrelated materials. Furthermore, the Kohn-Sham spectrum of this approach has proved to be an excellent starting point for calculations of physical excitation energies using perturbative approaches such as the GW approximation [2].

On the other hand, this approach has failed qualitatively in strongly-correlated systems such as Mott insulators, cuprates, manganites, and $f$-electron systems. Several directions have been pursued to treat such systems beyond LDA: The GW method [2], self-interaction corrected schemes [3], the $\mathrm{LDA}+\mathrm{U}$ method [4] and more recently $\mathrm{LDA}+\mathrm{DMFT}$ [5,6]. The $\mathrm{LDA}+\mathrm{U}$ approach combines the Dynamical Mean Field Theory (DMFT) [7] which has been very successful in the context of model Hamiltonians, with state-of-the-art band structure methods. This combination allows a coherent description of the electron in real and momentum space and leads to the appearance of both Hubbard bands and quasiparticle bands. Hence, it is well suited to describe strongly-correlated materials. For recent applications of this method, see [6,8,9].

In this Letter we introduce a new functional formulation of the electronic structure problem which leads to the LDA+DMFT equations and we present an application of the full implementation of this method to plutonium. In our generalization of DMFT to the electronic structure problem, our basic idea is to introduce another relevant variable in addition to the density $\rho$, namely the local Green function. The latter is defined by projecting the full Green function onto a separate subset of correlated "heavy" orbitals distinguished by the index $a$ from a complete set (indexed by $\alpha$ ) of orbitals $\chi_{\alpha}(\mathbf{r}-\mathbf{R}) \equiv \chi_{\alpha R}$ of a tight-binding representation which we assume for simplicity to be orthogonal. The local Green function is therefore given by a matrix $\hat{G}$ with elements [10]

$$
\begin{gathered}
G_{a b}(i \omega, R)=-\left\langle c_{a R}(i \omega) c_{b R}^{+}(i \omega)\right\rangle= \\
-\int \chi_{a}^{*}(\mathbf{r}-\mathbf{R})\left\langle\psi(\mathbf{r}, i \omega) \psi^{+}\left(\mathbf{r}^{\prime}, i \omega\right)\right\rangle \chi_{b}\left(\mathbf{r}^{\prime}-\mathbf{R}\right) d \mathbf{r} d \mathbf{r}^{\prime}
\end{gathered}
$$

We then construct a functional $\Gamma[\rho, \hat{G}]$ which gives the exact free energy at a stationary point. This generalizes the effective action construction used in connection with either pure DMFT functionals [10] or pure density functionals [11. It amounts to first considering the partition function of the interacting electron gas in the presence of a static source coupled to the density and a dynamic source coupled to the local spectral function, and second, carrying out a Legendre transformation with respect to those sources. This functional can be constructed formally in perturbation theory, however its explicit form is not available just as in density functional theory. The success of DMFT in model hamiltonians suggests a useful approximation to the exact functional, namely the LDA+DMFT functional, which allows us to compute both total energies and spectra.

To describe the new method, it is useful to introduce the notion of the Kohn-Sham potential $V_{K S}$ and its dynamical analog $\Sigma$. They are defined as the functions that one needs to add to the kinetic energy matrix so as to obtain a given density and spectral function of the heavy orbitals namely:

$$
\rho(\mathbf{r})=T \sum_{\omega} e^{i \omega 0^{+}}\left\langle\mathbf{r}\left|\left[i \omega+\nabla^{2} / 2-V_{K S}-\Sigma\right]^{-1}\right| \mathbf{r}\right\rangle .
$$

$V_{K S}$ is a function of $\mathbf{r}$, the chemical potential $\mu$ is set to zero throghout the paper, and $\Sigma$ is given by

$$
\Sigma \equiv \Sigma\left(\mathbf{r}, \mathbf{r}^{\prime}, i \omega\right)=\sum_{a b R} \chi_{a}^{*}(\mathbf{r}-\mathbf{R}) \Sigma_{a b}(i \omega) \chi_{b}\left(\mathbf{r}^{\prime}-\mathbf{R}\right),
$$

Recall that we are truncating to the "heavy" subset $\{a\}$ of orbitals. Then expression (2) becomes

$$
\rho(\mathbf{r})=T \sum_{a b} \sum_{\omega \mathbf{k}} \chi_{a \mathbf{k}}^{*}(\mathbf{r})\left[i \omega-\hat{H}^{\mathbf{k}}-\hat{\Sigma}(i \omega)\right]_{a b}^{-1} \chi_{b \mathbf{k}}(\mathbf{r}),
$$

where $H_{a b}^{\mathbf{k}}=\left\langle\chi_{a \mathbf{k}}\left|-\nabla^{2}+V_{K S}\right| \chi_{b \mathbf{k}}\right\rangle$ is the one-electron hamiltonian in $\mathbf{k}$-space. 
The physical meaning of the dynamical potential $\Sigma$ is parallel to the meaning of the original Kohn-Sham potential $V_{K S}$ : it is the function that one needs to add to the correlated block of the one-electron hamiltonian in order to obtain the desired local Green function:

$$
G_{a b}(i \omega)=\sum_{\mathbf{k}}\left[i \omega-\hat{H}^{\mathbf{k}}-\hat{\Sigma}(i \omega)\right]_{a b}^{-1} .
$$

It is the frequency dependence of the dynamical potential which allows us to treat Hubbard bands and quasiparticle bands on the same footing.

If the exact self energy of the problem is $\mathbf{k}$ independent, then it coincides with $\Sigma$. The parallel observation within DFT is: if the self energy of a model is $\mathbf{k}$ - and frequency-independent, then the self energy coincides with the Kohn-Sham potential.

In terms of these quantities, the external potential and the matrix of local interactions $\hat{U}$, we write down the DMFT+LDA functional:

$$
\begin{aligned}
& \Gamma_{L D A+D M F T}\left(\rho, V_{K S}, \hat{G}, \hat{\Sigma}\right)= \\
& -T \sum_{\omega} e^{i \omega 0^{+}} \operatorname{Tr} \log \left[i \omega+\nabla^{2}-V_{K S}-\Sigma\right] \\
& -\int V_{K S}(\mathbf{r}) \rho(\mathbf{r}) d \mathbf{r}-\sum_{\omega} e^{i \omega 0^{+}} \operatorname{Tr}[\hat{\Sigma}(i \omega) \hat{G}(i \omega)] \\
& +\int V_{e x t}(\mathbf{r}) \rho(\mathbf{r}) d \mathbf{r}+\frac{1}{2} \int \frac{\rho(\mathbf{r}) \rho\left(\mathbf{r}^{\prime}\right)}{\left|\mathbf{r}-\mathbf{r}^{\prime}\right|} d \mathbf{r} d \mathbf{r}^{\prime}+E_{x c}^{L D A}[\rho] \\
& +\sum_{R}\left[\Phi[\hat{G}]-\Phi_{D C}\right] .
\end{aligned}
$$

$\Phi[\hat{G}]$ is the sum of the two-particle irreducible local diagrams constructed with the local interaction matrix $\hat{U}$, and the local heavy propagator $\hat{G} \cdot \Phi_{D C}$ is the so-called double counting term which subtracts the average energy of the heavy level already described by LDA. We write it here in its simple form, when only one Slater integral is included, 1.e. $\Phi_{D C}=\bar{U} \bar{n}(\bar{n}-1) / 2$ with $\bar{n}=T \sum_{\omega, a b} G_{a b}(i \omega) e^{i \omega 0^{+}}$(see Ref. [4] for its general form). Expression Gensures that the Greens function obtained from its extremization will satisfy the Luttinger theorem.

An explicit form of $\Phi[\hat{G}]$ is unavailable. We can calculate it by relating it to the free energy of an atom in a medium, $W_{a t}$, which we describe by an action $S_{a t}$. To do this, we introduce the second central concept of DMFT: the Weiss field $G_{0}^{-1}$ which represents the quadratic part of the action $S_{a t}$.

$$
\begin{aligned}
S_{a t}\left[G_{0}^{-1}\right]= & \int_{\tau \tau^{\prime}} \sum_{a b} c_{a}^{+}(\tau)\left[G_{0}^{-1}\left(\tau, \tau^{\prime}\right)\right]_{a b} c_{b}\left(\tau^{\prime}\right)+ \\
& \int_{\tau} \sum_{a b c d} U_{a b c d} c_{a}^{+}(\tau) c_{b}^{+}(\tau) c_{c}(\tau) c_{d}(\tau) .
\end{aligned}
$$

Here, $\hat{G}_{0}^{-1}=\hat{G}_{a t}^{-1}-\hat{\Delta}$, where $\hat{G}_{a t}^{-1}$ describes the quadratic part of the action of the isolated atom, and $\hat{\Delta}$ describes the bath surrounding the atom. The bath added to the atomic action produces the desired local Green function i.e. $\left\langle c_{a} c_{b}^{+}\right\rangle_{S_{a t}}=G_{a b}$. We can now relate the sum of local graphs $\Phi[\hat{G}]$ to the free energy of the atom in the medium $W_{a t}=-\log \int \exp \left[-S_{a t}\right]$ via

$$
\Phi[\hat{G}]=W_{a t}\left[\hat{G}_{0}^{-1}\right]-\operatorname{Tr}\left(\hat{G}_{0}^{-1}-\hat{G}^{-1}\right) \hat{G}-\operatorname{Tr} \log \hat{G} .
$$

The functional (6) can be viewed as a functional of four independent variables, since the stationary condition in the conjugate fields reproduces the definition of the dynamical potential and the Weiss field. Extremizing it leads us to compute the Green function $G_{a b}(i \omega)$ Eq. (5) with the Kohn-Sham potential entering $H_{a b}^{\mathbf{k}}$ and with

$$
\Sigma_{a b}(i \omega)=\frac{\delta \Phi}{\delta G_{a b}(i \omega)}-\frac{\delta \Phi_{D C}}{\delta G_{a b}(i \omega)}
$$

which identifies $\Sigma(i \omega)$ as the self energy of a generalized Anderson impurity model [7] in a bath. The "impurity" is characterized by a matrix of (local) levels

$$
\epsilon_{a b}=\sum_{\mathbf{k}} H_{a b}^{\mathbf{k}}
$$

and is hybridized with the medium via the hybridization function $\Delta_{a b}(i \omega)$. The matrix $\delta \Phi_{D C} / \delta G_{a b}(i \omega)=$

$\bar{U}(\bar{n}-1 / 2)$ takes into account the double counting effects of the Coulomb interaction already contained in matrix $H_{a b}^{\mathbf{k}}$. The hybridization function $\Delta_{a b}(i \omega)$ obeys the selfconsistency condition

$$
\begin{aligned}
& i \omega-\epsilon_{a b}-\Delta_{a b}(i \omega)-\Sigma_{a b}(i \omega)= \\
& {\left[\sum_{\mathbf{k}}\left[i \omega-\hat{H}^{\mathbf{k}}-\hat{\Sigma}(i \omega)\right]^{-1}\right]_{a b}^{-1}}
\end{aligned}
$$

The self-consistent DMFT loop with respect to $\hat{\Delta}(i \omega)$ and $\hat{\Sigma}(i \omega)$ amounts to: (i) choosing some input hybridization $\hat{\Delta}(i \omega)$, (ii) solving the Anderson impurity model with that hybridization and extracting the impurity Green function and self energy $\hat{\Sigma}(i \omega)$, (iii) computing the on-site crystal Green function according to Eq. (5), and (iv) recovering a new hybridization function $\hat{\Delta}(i \omega)$ from Eq. (11).

Since the charge density depends on $\hat{\Sigma}(i \omega)$, another self-consistent loop is required to obtain the extremum of Eq. (6). This second loop updates the charge density once the DMFT loop delivers the self energy; this modifies the hopping integrals in the one-electron hamiltonian $\hat{H}^{\mathbf{k}}$. The new hoppings set up a new set of impurity levels $\epsilon_{a b}$. We see that the solution of eqs.(9)-(11) should be carried out in a double iterational loop: the DMFT loop finds $\hat{\Sigma}(i \omega)$ for given $\rho$ while the global one (DFT like) updates $\rho$. Within the DMFT technology, the implementation of this global loop is an essential new development 
of the present work which allows the determinatoion of a central quantity of the solid - its total energy.

We now illustrate the method applied to the electronic structure of $\mathrm{Pu}$ 12. Previous LDA 13,14 or LDA+U [15] or model [16] calculations have not been able to describe the existence of both a compressed $\alpha$ phase and a low-density $\delta$ phase, nor to provide a qualitative description of its photoemission spectra [17]. We have recently reported the energy vs volume curve and the one-electron spectrum for a value of $U$ which was estimated to correspond to that for $\delta \mathrm{Pu}$ [9]. Here, we study in detail the energy as a function of the parameter $U$ and give a comparative analysis of the spectra in both $\alpha$ and $\delta$ phases We also report calculations on a bcc structure corresponding to the higher temperature $\epsilon$ phase of $\mathrm{Pu}$.

We have implemented the spectral density functional approach described above using the linear muffin tin orbital (LMTO) method for electronic structure calculations. We used a generalized non-orthogonal tightbinding LMTO representation [18 which is most suited for describing the set of localized orbitals. The nonorthogonality corrections enter the definition for the Green function (5) in the form of an overlap matrix. To solve the impurity model for multi-orbital case of $\mathrm{Pu}$ we use a method which interpolates the self energy calculated from the Gutzwiller approximation [19] at low frequencies and a Hubbard-1-like approximation 20 at high frequencies [21]. Spin-orbit coupling effects are generally important for actinide compounds and have been included in the calculation for $\mathrm{Pu}$. The "full potential" terms have been neglected in the calculations through the utilization of the atomic sphere approximation with a one-kappa LMTO basis set 22].

The necessary $k$-space integrals for evaluating Green functions and charge densities have been carried out using the tetrahedron method with on a reciprocal grid $(8,8,8)$. The $\delta$ and $\epsilon$ phases of $\mathrm{Pu}$ have simple fcc and bcc structures respectively, while the $\alpha$ phase has a complicated lattice with 16 atoms per unit cell. We determined the ground state energy as a function of the atomic volume using fcc and bcc geometries. Finally, the approach requires the effective Coulomb interaction between $f$ electrons described by the Hubbard parameter $U$. Various estimates exist in the literature on the values of $U$ in plutonium which indicate that the average interaction among $f$ electrons is around $4 \mathrm{eV}$ [23.

We now report our calculated total energies for $\mathrm{Pu}$ in the fcc structure. The temperature is fixed at $600 \mathrm{~K}$, where the $\delta$ phase is stable. To illustrate the importance of correlations we perform the calculations for several values of $U$ varying it from 0 and $4 \mathrm{eV}$. The results are shown in Fig. 1: The $U=0$ (GGA) curve shows a minimum at $V / V_{\delta}=0.72$, close to the volume of the low-temperature $\alpha$ phase. Here $V_{\delta}$ is the observed $\delta$-phase volume. We expect that correlations should be less important for the compressed lattice in general, but there is no sign what- soever of the $\delta$ phase in the $U=0$ calculation. Dramatic changes occur when $U$ is about $4 \mathrm{eV}$. The curves show the possibility for a double minimum while the details depend sensitively on the actual value of $U$. We find that for $U=3.8 \mathrm{eV}$, the minimum occurs near $\mathrm{V} / \mathrm{V}_{\delta}=0.80$ which corresponds to the volume of the $\alpha$ phase. When $U$ increases by $0.2 \mathrm{eV}$ the minimum occurs at $\mathrm{V} / \mathrm{V}_{\delta}=1.05$ which corresponds to the volume of the $\delta$ phase, in close agreement with experiment. Clearly, since the energies are so similar, we may expect that as temperature decreases, the lattice undergoes a phase transition from the $\delta$ phase to the $\alpha$ phase with a remarkable decrease of the volume by $25 \%$.

We repeated our calculations for the bcc structure using the temperature $T=900 K$ where the $\epsilon$ phase is stable. Fig. 1 shows these results for $U=4 \mathrm{eV}$ with a location of the minimum at around $\mathrm{V} / \mathrm{V}_{\delta}=1.03$. While the theory has a residual inaccuracy in determining the $\delta$ and $\epsilon$ phase volumes by a few percent, a hint of volume decrease with the $\delta \rightarrow \epsilon$ transition is clearly reproduced. Thus, our first-principles calculations reproduce the main features of the experimental phase diagram of $\mathrm{Pu}$.

We now report our calculated spectral density of states for the fcc structure using the volumes $\mathrm{V} / \mathrm{V}_{\delta}=0.8$ and $\mathrm{V} / \mathrm{V}_{\delta}=1.05$ corresponding to the $\alpha$ and $\delta$ phases. Fig. 2 shows the results of our dynamical mean-field calculations as compared to the LDA-type calculations. We predict the appearance of a strong quasiparticle peak near the Fermi level which exists both in the $\alpha$ and $\delta$ phases. Recent advances have allowed the experimental determination of these spectra, and our calculations are in accord with these measurements [17]. The width of the quasiparticle peak in the $\alpha$ phase is found to be larger by 30 per cent compared to the width in the $\delta$ phase. This indicates that the low-temperature phase is more metallic, i.e. it has larger spectral weight in the quasiparticle peak and smaller weight in the Hubbard bands.

In conclusion, we have developed a new method for electronic structure calculations which allows the simultaneous evaluation of the total energy and the local electronic spectral density. We applied the method to study the phase diagram of $\mathrm{Pu}$ and have reproduced several of its salient features. While we have not yet included the electronic and phonon entropy terms which are difficult to evaluate numerically, many properties of realistic strongly correlated systems which are related to the total energy can now be explored using LDA+DMFT.

The authors are indebted to E. Abrahams, A. Arko, J. Joyce and J. Thompson for helpful discussions. This research was supported by the US DOE, grant No. DEFG02-99ER45761, by Los Alamos National Laboratory under subcontract No. 25124-001-012K, and by the NSF DMR-0096462. 
[1] For a review, see e.g., Theory of the Inhomogeneous Electron Gas, edited by S. Lundqvist and S. H. March (Plenum, New York, 1983);

[2] For a review, see, F. Aryasetiawan and O. Gunnarsson, Rep. Prog. Phys. 61, 237 (1998);

[3] See e.g., A. Svane and O. Gunnarsson, Phys. Rev. Lett. 65, 1148 (1990); Z. Szotek, W. M. Temmerman and H. Winter, Phys. Rev. B47, 4029 (1993);

[4] For a review, see, V. I. Anisimov, F. Aryasetiawan, and A. I. Liechtenstein, J. Phys. Cond. Matter 9, 767 (1997);

[5] V. I. Anisimov, A. I. Poteryaev, M. A. Korotin, A. O. Anokhin, and G. Kotliar, J. Phys.: Condens. Matter 35, 7359 (1997); A. Lichtenstein and M. Katsenelson, Phys. Rev. B57, 6884 (1998);

[6] A. Lichtenstein and M. Katsnelson, Phys. Rev. B 57, 6884 (1998);

[7] A. Georges, G. Kotliar, W. Krauth, and M. J. Rozenberg, Rev. Mod. Phys. 68, 13 (1996);

[8] I.A. Nekrasov, K. Held, N. Blumer, A.I. Poteryaev, V.I. Anisimov, and D. Vollhardt, Eur. Phys. J. B18, 55 (2000);

[9] S. Savrasov, G. Kotliar, and E. Abrahams, Nature 410, 793 (2001);

[10] R. Chitra and G. Kotliar, Phys. Rev. B62, 12715 (2000);

[11] R. Fukuda, T. Kotani, and S. Yokojima, Prog. Theor. Phys. 92, 833 (1994); R. Fukuda et al, Prog. Theor. Phys. Suppl. 121, 1 (1996); M. Valiev and G. Fernando, Phys. Lett. A227, 265 (1997);

[12] For a review, see e.g., The Actinides: Electronic Strucuture and Related Properties, edited by A. J. Freeman and J. B. Darby, Vols. 1 and 2 (Academic Press, New York, 1974);

[13] For recent calculations, see, P. Söderlind, O. Eriksson, B. Johansson, and J. M. Wills, Phys. Rev. B50, 7291 (1994); M. D. Jones, J. C. Boettger, R. C. Albers, and D. J. Singh, Phys. Rev. B61, 4644 (2000);
[14] I. V. Solovyev, A. I. Liechtenstein, V. A. Gubanov, V. P. Antropov, and O. K. Andersen, Phys. Rev. B43, 14414 (1991);

[15] S. Savrasov and G. Kotliar, Phys. Rev. Lett. 84, 3670 (2000); J. Bouchet, B. Siberchicot, F. Jollet, and A. Pasturel, J. Phys.: Condens. Matter 12, 1 (2000);

[16] O. Eriksson, J. D. Becker, A. V. Balatsky, and J. M. Wills, J. Alloys and Comp. 287, 1 (1999);

[17] A.J. Arko, J.J. Joyce, L. Morales, J. Wills, and J. Jashley, Phys. Rev. B62, 1773 (2000);

[18] O. K. Andersen and O. Jepsen, Phys. Rev. Lett. (1984);

[19] M. Gutzwiller, Phys. Rev. (1961);

[20] J. Hubbard, Proc. Roy. Soc. (London) A281, 401 (1964);

[21] S. Y. Savrasov, G. Kotliar, D. Villani, and V. Udovenko, (unpublished);

[22] S. Y. Savrasov, Phys. Rev. B54, 16470 (1996);

[23] See e.g., J. P. Desclaux and A. J. Freeman, in Handbook on the Physics and Chemistry of the Actinides, edited by A. J.. Freeman and G. H. Lander (Elsevier, Amsterdam, 1984), Vol. 1;

\section{FIGURE CAPTIONS}

Fig. 1. Total energy as a function of volume in $\mathrm{Pu}$ for different values of $U$ calculated using the LDA+DMFT approach. Data for the fcc lattice are computed at $\mathrm{T}=600 \mathrm{~K}$, while data for the bcc lattice are given for $\mathrm{T}=900 \mathrm{~K}$.

Fig. 2. Comparison between photoemission spectra of $\delta-\mathrm{Pu}$ [17 (circles) and calculated densities of states using the LDA+DMFT approach: the data for $V / V_{\delta}=1.05, U=4.0 \mathrm{eV}$ (full line) and the data for $V / V_{\delta}=0.80, U=3.8 \mathrm{eV}$ (dashed line) correspond to the volumes of the $\delta$ and $\alpha$ phases respectively. The result of the GGA calculation (dotted line) at $V / V_{\delta}=1(U=0)$ is also given. 
Fig. 1, Savrasov et. al

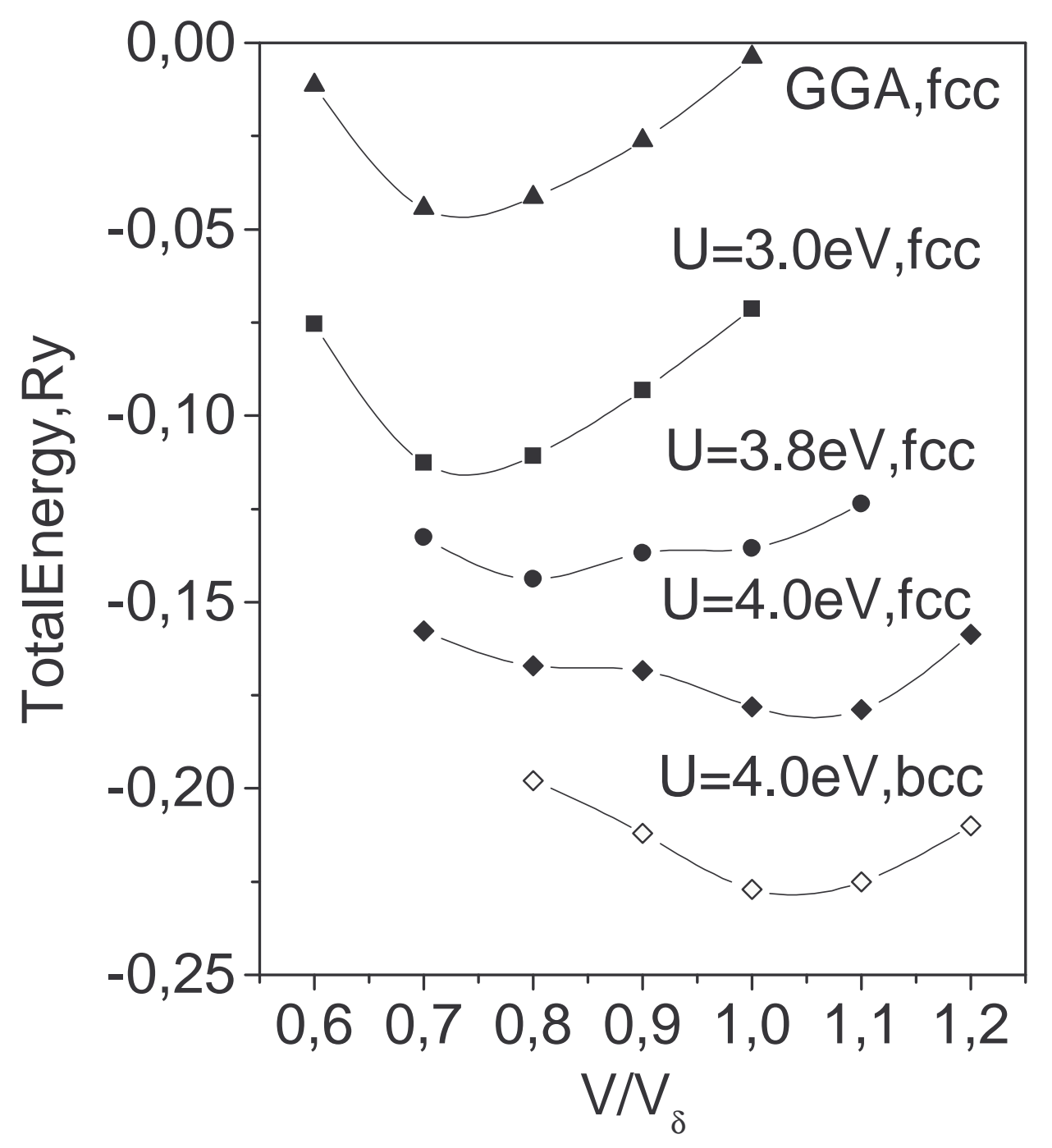


Fig. 2, Savrasov et.al

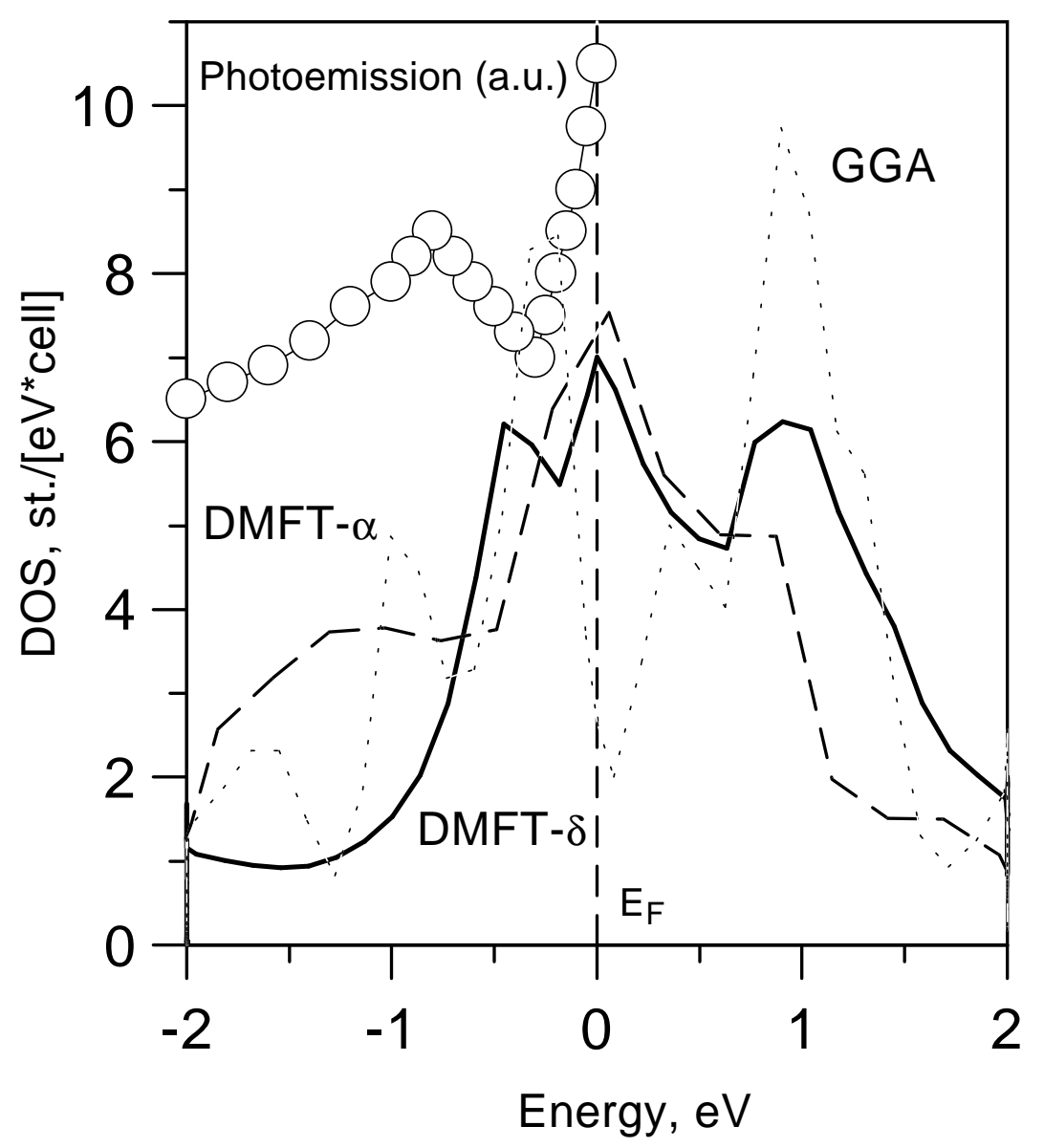

